\title{
Multicriteria Fuzzy Sets Application in Economic Clustering Problems
}

\section{Irina Radeva}

Institute of Information and Communication Technologies, Bulgarian Academy of Sciences, 1113 Sofia, Bulgaria

E-mail:iradeva@iit.bas.bg

\begin{abstract}
This paper presents an approach for small and medium-sized enterprises selection in economic clusters, where the problem of integration is defined as "ill structured under condition of uncertainty". The proposed solution demonstrates applying several fuzzy multi-criteria decision making algorithms along with discussion over specific input data requirements. The results are compared with classical multi-criteria decision-making algorithm PROMETHEE II.
\end{abstract}

Keywords: Multi-criteria decision, fuzzy sets algorithms, intercriteria analysis, small and medium-sized enterprises, economic clustering.

\section{Introduction}

The majority of measures to stimulate competitiveness in economy to a large extent are focused on management and cooperation of small and medium-sized enterprises $[4,24]$. One of the effective recommended polices is economic clustering. Studies in this area are mainly focused on regional and product integration. The results are related to identifying naturally occurring clusters, studying their effectiveness and expansion, through top-down management actions [23]. Economic cluster is a group of enterprises joined by stable economic, political and innovation social relations, which are not defined by an organised membership. The strategic purpose is increasing the degree of knowledge and establishing new networks of communication in the production of innovative products [25].

When developing decision-making tools for cluster design, some of the approaches are based on multi-criteria methods working in a determined [5] and/or fuzzy environment. Researches related to the development of tools for clusters design has traditionally based on determinate information. Here is presented an attempt to solve decision-making problems using an approach based on methods of fuzzy sets theory since it gains popularity in variety of applications [10-13,27]. The problem of selection of cluster structure design can be classified as a "poorly structured" task under uncertainty. 
In the paper is proposed an approach for initial step of economic clustering that allows selected list of enterprises to be ranked in a descending order of their integrated development status assessments. The aim is to include them in a certain technological network node of the cluster by using multi-criteria decision methods under conditions of determinism or fuzziness. The suggested decision is based on descending ranking of integral score of enterprises' business performance within nodes of predetermined Technological Network (TN).

The tests are performed using software designs of fuzzy models [15-21] presented and tested in [6]. The solutions are based on several groups of algorithms classified as follows:

A. Criteria - real numbers, fuzzy relations and/or fuzzy numbers

- Weighting coefficients - real numbers: Algorithm with aggregation operators with weighting coefficients real numbers (ATOKRI1) and algorithm with aggregation operators without weighting coefficients for criteria (ATOKRI2);

- Weighting coefficients - fuzzy relations: Algorithm with ranking function (ARAKRI1) and mixed data algorithm (ARAKRI3);

- Weighting coefficients - real functions: Algorithm for crisp criteria with weighted coefficients - weighting functions (ATOKRIF).

B. Criteria - only fuzzy numbers.

C. No weighting coefficients - direct aggregation fuzzy numbers algorithm (ARAKRI2).

The implementation of presented approach is illustrated by an example performed by above fuzzy algorithms and results are compared to classical multicriteria outranking method PROMETHEE II [3].

\section{The economic clustering problem definition}

The economic clustering problem on a given technology network is defined as a multi-criteria selection task and is partially described in [25, 26].

There are three tasks defined as:

Task 1. Preparing of a list of enterprises - potential participants in the cluster with an indication of their membership to a particular node of TN.

Task 2. Developing of a "passport" for each enterprise, according to a predetermined system of criteria.

Task 3. Assessing and ranking of enterprises scores in decreasing order on TN nodes. Based on this decision, selection of lists of potential participants for each node of TN.

Diagram of the approach is shown in Fig. 1. Tasks 1 and 2 carry out the preparatory phase. Task 3 implements the analytical and decision-making stage.

Task 1 is considering four steps.

Step 1. Description and setting the number of nodes $t$ in TN (branch affiliation, and relations in technological chain of production).

Step 2. Setting lists of enterprises ${ }_{n}$, where $n$ indicates the number of potential participants. Such list is prepared for each node $t$. 
Step 3. Developing the structure of a "passport" for each node $t$. Assessments are performed for enterprises by nodes $t$ individually.

Step 4. Collecting, selecting and processing "passport" data. (For the purposes of this study, a universal "passport" structure is adopted for all $t$ nodes.)

Task 2 consists three steps.

Step 1. Determination of ranking lists according to the number of nodes $t$.

Step 2. Determination of number of alternatives and criteria. The number of alternatives depends on the number of enterprises. A "passport" determines the number of criteria.

Step 3. Processing the primary information and selection of the type of input data.

Task 3 is analysis of rankings and final selection of enterprises.

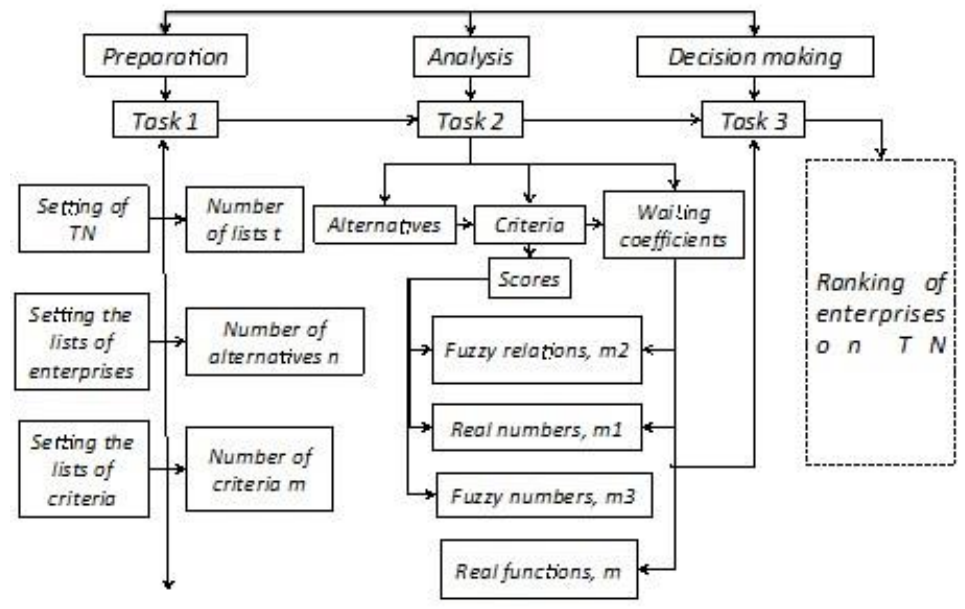

Fig. 1. Diagram of economic clustering approach

\section{Data definitions}

A finite set of alternatives $A=\left\{a_{1}, \ldots, a_{j}, \ldots, a_{n}\right\}$ is defined, evaluated by criteria $K=\left\{k_{1}, \ldots, k_{j}, \ldots, k_{m}\right\}$. The weights of criteria are $W=\left\{w_{1}, \ldots, w_{j}, \ldots, w_{m}\right\}$. The final set $A$ is the list of enterprises in a given node. The final set $K$ contains Passport's criteria. The input data are presented in Table 1. The number of tables equals the number of technological nodes in a cluster.

The real numbers $x_{i j}, i=1, \ldots, n ; j=1, \ldots, m$, in the table are the assessment of the alternative $a_{i}$ by the criterion $k_{j}$. To each criterion $k_{j}$ is assigned a weighting coefficient $w_{j}$. The total number of selected criteria equals to $m$, such as $m=m_{1}+$ $m_{2}+m_{3}$, where: $m_{1}$ is the number of criteria that are set as crisp numbers, $m_{2}$ is the number of criteria that are set as fuzzy relations, and $m_{3}$ is the number of criteria that are given as fuzzy numbers. 
Table 1. The input data

\begin{tabular}{|c|c|c|c|c|c|}
\hline \multirow{2}{*}{ Alternatives } & \multicolumn{5}{|c|}{ Criteria } \\
\cline { 2 - 6 } & $k_{1}$ & $\ldots$ & $k_{j}$ & $\ldots$ & $k_{m}$ \\
\hline$a_{1}$ & $x_{11}$ & $\ldots$ & $x_{1 j}$ & $\ldots$ & $x_{1 m}$ \\
\hline$\vdots$ & $\vdots$ & $\vdots$ & $\vdots$ & $\vdots$ & $\vdots$ \\
\hline$a_{i}$ & $x_{i 1}$ & $\ldots$ & $x_{i j}$ & $\ldots$ & $x_{i m}$ \\
\hline$\vdots$ & $\vdots$ & $\vdots$ & $\vdots$ & $\vdots$ & $\vdots$ \\
\hline$a_{n}$ & $x_{n 1}$ & $\ldots$ & $x_{n j}$ & $\ldots$ & $x_{n m}$ \\
\hline Weights of criteria & $w_{1}$ & $\ldots$ & $w_{j}$ & $\ldots$ & $w_{m}$ \\
\hline
\end{tabular}

\section{Data presentation and different cases}

The combinations of data determine algorithms in use. The criteria data, weighting coefficients, and algorithms used are systematized in Table 2.

Table 2. Criteria data, weighting coefficients and algorithms

\begin{tabular}{|c|c|c|c|c|c|}
\hline \multicolumn{2}{|c|}{ Criteria data } & Non (w) & $\begin{array}{l}\text { (w) Real } \\
\text { numbers }\end{array}$ & $\begin{array}{l}\text { (w) Fuzzy } \\
\text { relations }\end{array}$ & $\begin{array}{l}\text { (w) Real } \\
\text { functions }\end{array}$ \\
\hline \multirow{3}{*}{$\begin{array}{l}\text { One type } \\
\text { criteria }\end{array}$} & $\begin{array}{c}\text { Real } \\
\text { numbers } m_{1}\end{array}$ & ATOKRI2 & $\begin{array}{l}\text { ATOKRI1 or } \\
\text { ATOKRI2 }\end{array}$ & $\begin{array}{c}\text { ATOKRI1 -> } \\
\text { ARAKRI }\end{array}$ & $\begin{array}{c}\text { ATOKRI -> } \\
\text { ATOKRIF }\end{array}$ \\
\hline & $\begin{array}{l}\text { or fuzzy } \\
\text { relations } m_{2}\end{array}$ & & $\begin{array}{c}\text { ATOKRI1 or } \\
\text { ATOKRI2 }\end{array}$ & ARAKRI & ATOKRIF \\
\hline & $\begin{array}{c}\text { or fuzzy } \\
\text { numbers } m_{3}\end{array}$ & & $\begin{array}{c}\text { ARAKRI } 1 \text { or } \\
\text { ARAKRI } 2\end{array}$ & & $\begin{array}{c}\text { ARAKRI1 } \\
\text { or } \\
\text { ARAKRI2 } \\
\end{array}$ \\
\hline \multirow{3}{*}{$\begin{array}{l}\text { Mixed type } \\
\text { criteria }\end{array}$} & $\begin{array}{c}\text { Real } \\
\text { numbers } m_{1}\end{array}$ & & $\begin{array}{l}\text { ARAKRI1, } \\
\text { ATOKRI1 or } \\
\text { ATOKRI2 }\end{array}$ & $\begin{array}{l}\text { ATARR, } \\
\text { ARAKRI1 }\end{array}$ & $\begin{array}{l}\text { ARAKRI1, } \\
\text { MMTRR, } \\
\text { ATOKRIF }\end{array}$ \\
\hline & $\begin{array}{c}\text { Fuzzy } \\
\text { relations } m_{2}\end{array}$ & & MMTRR & & \\
\hline & $\begin{array}{c}\text { Fuzzy } \\
\text { numbers } m_{3}\end{array}$ & & ARAKRI3 & & \\
\hline
\end{tabular}

A block-diagram of algorithms application is given in Fig. 2.

Case 1. Crisp criteria assessment and crisp weights

All criteria - crisp $\left(m_{1}=m\right)$ and weighting coefficients - real numbers $\left(w_{1}, \ldots, w_{m}\right)$. The crisp criteria assessments are limited to fuzzy relations with certain properties. The calculations $[22,28]$ are using aggregation operator algorithm ATOKRI1 or algorithm with aggregation operator without weighting coefficients ATOKRI2, where transformations of ATOKRI1 fuzzy relations generated by ATOKRI1 are performed and then aggregation operators without weighting coefficients are used. 


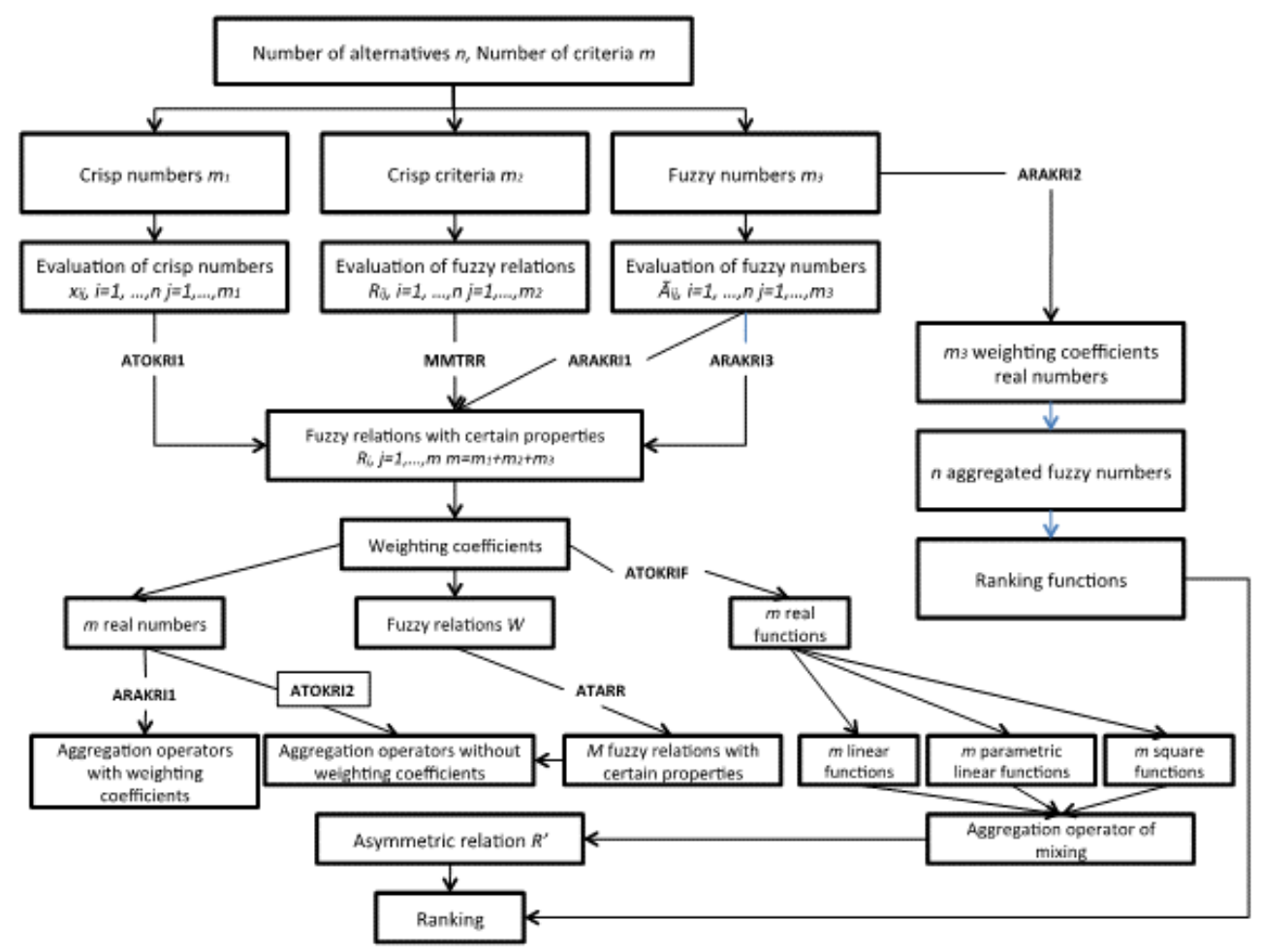

Fig. 2. Block-diagram of fuzzy algorithms application

The algorithm ATOKRI1 uses data from Table 1. Since criteria assessments can be set in different measuring scales it is necessary to normalize

$$
\mu_{k}\left(a_{i}, a_{j}\right)= \begin{cases}1 & \text { if } i=j, \\ 0.5+\frac{x_{i k}-x_{j k}}{2\left(\max _{i}\left\{x_{i k}\right\}-\min _{i}\left\{x_{i k}\right\}\right.} & \text { if } i \neq j,\end{cases}
$$

where $\mu_{k}\left(a_{i}, a_{j}\right), \quad a_{i}, a_{j} \in A, k \in\left\{k_{1}, k_{2}, \ldots, k_{m}\right\}$.

For each column of Table 1 the corresponding fuzzy relation $R_{k}$ is calculated, i.e., for $k$-criteria. Corresponding fuzzy relation in the matrix is

$$
R_{k}=\left\{\mu_{k}\left(a_{1}, a_{j}\right\}, i, j=1, \ldots, n, k=1, \ldots, m .\right.
$$

The minimizing criteria should be transformed into maximizing.

Relations $R_{k}$ are shrunk to aggregated relations $R$ with the following matrix:

$$
R=\left\{\mu_{k}\left(a_{1}, a_{j}\right)\right\}, i, j=1, \ldots, n .
$$

The matrix elements are computed by aggregation operators with weighting coefficients $\left\{w_{1}, \ldots, w_{j}, \ldots, w_{m}\right\}$. The following operators are used and values for $\mu_{k}\left(a_{i}, a_{j}\right)$ are derived form (1):

$$
\begin{gathered}
\mu\left(a_{i}, a_{j}\right)=\sum_{k=1}^{m} w_{k} \mu_{k}\left(a_{i}, a_{j}\right), \text { where } 0 \leq w_{k} \leq 1, \sum_{k=1}^{m} w_{k}=1, \\
\mu\left(a_{i}, a_{j}\right)=\prod_{k=1}^{m}\left[\mu_{k}\left(a_{i}, a_{j}\right)\right]^{w_{k}}, \text { where } 0<w_{k i} \leq 1, \sum_{k=1}^{m} w_{k}=1 .
\end{gathered}
$$


For two operators above weights of the criteria are normalized:

$$
\begin{gathered}
\mu\left(a_{i}, a_{j}\right)=\max _{k}\left\{\min \left(\mu_{k}\left(a_{i}, a_{j}\right), w_{k}\right)\right\}, \text { where } 0 \leq w_{k} \leq 1, \max _{k i}\left\{w_{k}\right\}=1, \\
k=1, \ldots, m, \\
\mu\left(a_{i}, a_{j}\right)=\min _{k}\left\{\max \left(\mu_{k}\left(a_{i}, a_{j}\right), 1-w_{k}\right)\right\}, \text { where } 0 \leq w_{k} \leq 1, \max _{k i}\left\{w_{k}\right\}=1, \\
k=1, \ldots, m .
\end{gathered}
$$

For two operators above weights of the criteria are also normalized.

There are obtained four aggregated relations, i.e., four matrices of type $R(2)$. Each of matrices is recalculated to matrices $R^{\prime}$ in the following manner:

(7) $\quad$ if $\mu\left(a_{i}, a_{j}\right) \geq \mu\left(a_{j}, a_{i}\right)$, then $\mu^{\prime}\left(a_{i}, a_{j}\right)=\mu\left(a_{i}, a_{j}\right)$ and $\mu^{\prime}\left(a_{j}, a_{i}\right)=0$.

The matrices $R^{\prime}$ are rearranged to triangular matrices that show the order of alternatives in descending order depending on selected aggregation operator.

The difference between ATOKRI2 and ATOKRI1 is in the choice of aggregation operators. ATOKRI2 uses operators without weighing coefficients. If the criteria weights are not set, then after obtaining the fuzzy relations $R_{k}, k=1, \ldots, m$, by (1) and recalculation of the minimizing criteria from the corresponding steps of ATOKRI1, aggregated relations are calculated by aggregating operators:

$$
\text { (10) } \mu\left(a_{i}, a_{j}\right)=\left\{\begin{array}{l}
{\left[\prod_{k=1}^{m} \mu\left(a_{i}, a_{j}\right)\right]^{1-\gamma}\left[1-\prod_{k=1}^{m}\left(1-\mu\left(a_{i}, a_{j}\right)\right)\right]^{\gamma} \text { if } \mu\left(a_{i}, a_{j}\right) \neq 0 .} \\
0 \quad \text { otherwise. }
\end{array}\right.
$$$$
\mu\left(a_{i}, a_{j}\right)=\alpha \max \left\{\mu_{k}\left(a_{i}, a_{j}\right)\right\}+(1-\alpha) \min \left\{\mu_{k}\left(a_{i}, a_{j}\right)\right\},
$$$$
\alpha \in[0,1], i, j=1, \ldots, n, k=1, \ldots m \text {; }
$$

The values $\mu_{k}\left(a_{i}, a_{j}\right)$ are taken from (1). Coefficients $\alpha, \lambda, \gamma$ are set according to particular task. It can be experimented with different values.

Since aggregated relations (aggregated matrices of type $R(2)$ ) are obtained then the other steps of ATOKRI1 algorithm are followed, i.e., each matrix is recalculated to $R^{\prime}$ matrices by (7). The $R^{\prime}$ matrices are ranked and produce triangular matrices that show descending order of alternatives depending on selected aggregation operator. In order to use the above aggregation operators, if criteria weights are set, the steps of the algorithm are following: the values of the matrices $R_{k}, k=1, \ldots, m$, by (1), are transformed:

(11) $\mu_{k}^{1}\left(a_{i}, a_{j}\right)=\left(1-w_{k}\right)+\mu_{k}\left(a_{i}, a_{j}\right)-\left(1-w_{k}\right) \mu_{k}\left(a_{i}, a_{j}\right), i, j=1, \ldots, n$, or

$$
\mu_{k}^{2}\left(a_{i}, a_{j}\right)=w_{k} \mu_{k}\left(a_{i}, a_{j}\right)
$$

where $\mu_{k}\left(a_{i}, a_{j}\right)$ is a corresponding element of the matrix (1).

For each relation $R_{k}, k=1, \ldots, m$, is obtained two matrices $R_{k}^{1}=\left\{\mu_{k}^{1}\left(a_{i}, a_{j}\right\}\right.$, $R_{k}^{2}=\left\{\mu_{k}^{2}\left(a_{i}, a_{j}\right\}\right.$. For $R(2)$ are used aggregation operators (8), (9), (10) for respective aggregated relations. Calculations proceed by following the other steps of ATOKRI1 algorithm (see Fig. 2). 
Case 2. Crisp criteria assessments without weighing coefficients

All criteria are crisp, $m_{1}=m$. Criteria weights are not specified - the crisp estimates of criteria are reduced to fuzzy relations with certain properties and calculations are performed with ATOKRI2 (see Fig. 2).

Case 3. Ccrisp criteria assessments and weighting coefficients - real functions

All criteria are crisp, $m_{1}=m$. Weighting coefficients are real functions $f_{1}(x), \ldots, f_{m}(x), x \in[0,1]$ - crisp criteria estimates are reduced to fuzzy relations using ATOKRI1 algorithm, then ATOKRIF is used for crisp criteria with weighting coefficients - weighting functions.

The difference between ATOKRIF and ATOKRI algorithms is in the choice of weighting coefficients for criteria. ATOKRIF uses as weighting coefficients weighting functions $f_{1}(x), \ldots, f_{m}(x), x \in[0,1]$, with arguments the elements of corresponding matrices (1).

The input data for ATOKRIF are fuzzy relations. If relations are derived from ATOKRI1, they have the proper properties to obtain a ranking. If relations are set, they must be checked for required properties. If requirements are not met, they are transformed into respective relations. So, the relations are processed with MMTRR algorithm. Let obtained from ATOKRI1 relations are $R_{k}, k=1, \ldots, m$. Aggregated relations are calculated by selection of one of the following weighing functions. The setting of relevant function parameters that must meet conditions:

- Linear functions

- Parametric linear functions

$$
\begin{gathered}
f_{k}(x)=a_{k} \frac{1+\beta_{k} x}{1+\beta_{k}}=\gamma_{k}\left(1+1+\beta_{k} x\right) . \\
0 \leq \alpha_{k} \leq 1,0 \leq \beta_{k} \leq 1, \gamma_{k}=\frac{\alpha_{k}}{1+\beta_{k}}, k=1, \ldots, m .
\end{gathered}
$$

- Quadratic functions

(15) $f_{k}(x)=1+\left(\beta_{k}-\gamma_{k}\right) x+\gamma_{k} x^{2}, \beta_{k} \geq 0, \gamma_{k} \geq 0, k=1, \ldots, m$.

Function's parameters are unrelated and different for different functions.

For each relation $R_{k}, k=1, \ldots, m$ a new degree of preference for each criteria $k$ are calculated with corresponding weighting function:

$$
\mu_{k}^{w}\left(a_{i}, a_{j}\right)=\left\{\begin{array}{ll}
1 & \text { if } a_{i}=a_{j} \\
\frac{f_{k}\left(\mu_{k}\left(a_{i}, a_{j}\right)\right) \mu_{k}\left(a_{i}, a_{j}\right)}{S\left(a_{i}, a_{j}\right)} & \text { if } a_{i} \neq a_{j}
\end{array}, \quad i, j=1,2,3, \quad k=1,2,\right.
$$

where $S\left(a_{i}, a_{j}\right)=\sum_{k=1}^{m} f_{k}\left(\mu_{k}\left(a_{i}, a_{j}\right)\right), f_{k}($.$) is one of weighting functions$ (13)-(15).

The result is $m$ matrices $R_{k}^{w}=\left\{\mu_{k}^{w}\left(a_{i}, a_{j}\right\}, i, j=1, \ldots, n, k=1, \ldots, m\right.$.

These matrices are combined into a matrix of the type $\mathrm{R}(2)$, the elements of which are obtained by sum of corresponding elements of matrices $R_{k}^{w}$. Based on the $R$-type matrix, other steps of ATOKRI1 algorithm are followed (see Fig. 2), i.e., recalculation to the matrices $R^{\prime}$ by (7). The resulting matrix $R^{\prime}$ is reordered to obtain a triangular matrix that shows descending ranking of alternatives. 
Case 4. Crisp criteria assessments and fuzzy relation of preference for criteria weights

All criteria are crisp $m=m_{1}$. A fuzzy relation of preference $W$ is set for criteria weight - the crisp criteria estimates are reduced to fuzzy relations with ATOKRI1. Then ARAKRI is used for fuzzy relations of alternatives and weights of criteria. In ARAKRI the input data are fuzzy relations of type (1) between alternatives by each criterion and a fuzzy relation between criteria. The input data for criteria can be both crisp (as in Table 1) and fuzzy relations of type (1). For crisp criteria, the first steps of ATOKRI1 algorithm calculate fuzzy relations of alternatives. If criteria assessments are set directly as fuzzy relations, then it is checked for additive transitivity properties of reciprocal relations. If not, the initial relations are transformed (using ATARR algorithm described in Case 7) into new fuzzy relations with necessary properties. The weighting coefficients of criteria are given as a fuzzy relation with certain properties of:

$$
W=\left\{\begin{array}{ll}
0.5, & i=j \\
1-w_{i, j}, & i \neq j
\end{array}, i, j=1, \ldots, m .\right.
$$

After receiving the matrices by all criteria $R_{k}, k=1, \ldots, m$, i.e., one obtained from ATOKRI1 and the other verified for required properties, there are given two possibilities for joining each matrix pair to include the elements of the matrix $W$. If the fuzzy relations are consistent with criteria $R_{1}, \ldots, R_{m}$, then by merging $R_{i}$ and $R_{j}$, taking into account respective elements of the matrix $\mathrm{W}$ it is obtained a new relation $R_{i j}$ and since $R_{i j}=R_{j i}$, the number $p$ of the new relation will be equal to the combination of two elements of $m$, i.e., $p=\frac{m(m-1)}{1.2}$. For aggregation of these new pairs $p$, ATOKRI2 algorithm is used and the next steps are proceed (Fig. 2).

Case 5. Criteria evaluations - fuzzy relations, weighting coefficients are real numbers

All criteria set fuzzy relations of preference between alternatives, $m_{2}=m$ and weighting coefficients are real numbers. Fuzzy relations are checked for max-min transitivity (using MMTRR algorithm). Those that are not are transformed into new relations. ATOKRI1 with aggregation operators with weighted coefficients is used and/or ATOKRI2 where weight transformations of fuzzy relations are performed. Then aggregation operators without weighting coefficients are used. The algorithm MMTRR includes:

A. Verification algorithm for max-min transitivity of two relations:

1. It is given a relation $R=\left\|r_{i j}\right\|, r_{i j}=1, i, j=1, \ldots, n$.

2. If $r_{i j} \geq \min \left(r_{i k}, r_{k j}\right), \forall i, j, k=1, \ldots, n$, relation is max-min transitive, otherwise it is transformed into max-min transitive relation using the following algorithm.

B. Algorithm for obtaining a max-min transitive relation:

1. It is given a relation $R=\left\|r_{i j}\right\|, i, j=1, \ldots, n$.

2. Calculate:

$$
R^{2}=R \circ R=\left\|r_{i j}^{2}\right\|, r_{i j}^{2}=\max \left\{\min \left(r_{i k}, r_{k j}\right\}, i, j, k=1, \ldots, n,\right.
$$

$$
R^{3}=R^{2} \circ R=\left\|r_{i j}^{3}\right\|, \quad r_{i j}^{3}=\max \left\{\min \left(r_{i k}^{2}, r_{k j}\right\}, \quad i, j, k=1, \ldots, n .\right.
$$


3. For a matrix of $n \times n$ dimensions, $n$ is the number of matrices, where $R^{i}=R^{i-1} \circ R, i \geq 2 R^{i}=\left\|r_{i j}^{i}\right\|, r_{i j}^{i}=\max \left\{\min \left(r_{i k}^{i-1}, r_{k j}\right\}, i, j, k=1, \ldots, n\right.$.

4. The transitive closing of $R$ is a transitive fuzzy relation that contains $R_{T}$ and $R_{T}=R \cup R^{2} \cup \ldots \cup R^{n}$.

Case 6. Criteria assessments - fuzzy relation, weighting coefficients are real functions

All criteria are set as fuzzy relations of preference between alternatives, i.e., $m_{2}=m$. Weighting coefficients are real functions $f_{1}(x), \ldots, f_{m}(x), x \in[0,1]$. Using MMTRR algorithm fuzzy relations are checked for certain properties. Then the steps of ATOKRIF are proceeding.

Case 7. Criteria assessments and weighting coefficients are fuzzy preference relations

All criteria are set as fuzzy relations of preference between alternatives, i.e., $m_{2}=m$. Fuzzy preference relation $W$ (weights) of criteria is given. Fuzzy relations are checked for the additive transitivity of reciprocal relations with ATARR algorithm [12]. The next steps of ARAKRI are proceeding (Fig. 2).

Case 8. Criteria assessments - fuzzy numbers and weighting coefficients are real numbers

All criteria give fuzzy numbers for evaluations of alternatives, i.e., $m_{3}=m$ and weighting coefficients are real numbers $w_{1}, \ldots, w_{m}$. One or more algorithms ARAKRI are used when evaluating the fuzzy numbers. For these algorithms, it is required estimates of alternatives for all criteria to be fuzzy or real numbers. Two approaches are then proposed for decision-making. One is ARAKRI1 algorithm, where fuzzy numbers are replaced by a corresponding real index by a ranking function. The aggregation is by aggregating operators depending on set weights of criteria. The other is ARAKRI2 algorithm where no ranging function is used, but aggregating operators directly generates aggregated fuzzy numbers. The advantage of ARAKRI2 is the smaller amount of input data needed. As this method does not require weighting coefficients, memory complexity is reduced and expert's subjectivity is substantially less [6].

Evaluations of the alternatives according to criteria in ARAKRI1 are fuzzy numbers given in a matrix form

$$
\left[\begin{array}{cccc} 
& k_{1} \ldots . . & k_{j} \ldots \ldots & k_{m} \\
a_{1} & \tilde{A}_{11} & \tilde{A}_{1 j} & \tilde{A}_{1 m} \\
\vdots & \vdots & \vdots & \vdots \\
a_{i} & \tilde{A}_{i 1} & \tilde{A}_{i j} & \tilde{A}_{i m} \\
\vdots & \vdots & \vdots & \vdots \\
a_{n} & \tilde{A}_{n 1} & \tilde{A}_{n j} & \tilde{A}_{n m}
\end{array}\right],
$$

where the fuzzy numbers are of the type: $\tilde{A}_{i j}=\left(a_{i j}^{1}, a_{i j}^{2}, a_{i j}^{3}, a_{i j}^{4}\right), i=1, \ldots, n$, $j=1, \ldots, m, a_{i j}^{1} \leq a_{i j}^{2} \leq a_{i j}^{3} \leq a_{i j}^{4}$ are real numbers and for corresponding criteria these assessments could be of different scales and unification and normalization of fuzzy numbers is needed. The unified and normalized fuzzy number $\tilde{Z}_{i j}=\left(z_{i j}^{1}, z_{i j}^{2}, z_{i j}^{3}, z_{i j}^{4}\right)$, is estimated by formula 


$$
\frac{\tilde{Z}_{i j}=\left(\tilde{A}_{i j}-a_{j}^{\min }\right)}{d a}, i=1, \ldots, n, j=1, \ldots, m .
$$

If some of criteria are maximizing and some minimizing, then for the minimization of criteria the additions of the fuzzy numbers to fuzzy number $(1,1,1,1)$ are calculated. The unified and normalized fuzzy indexes $F\left(\tilde{Z}_{i j}\right)$ are calculated using the ranging function

$$
F\left(\tilde{Z}_{i j}\right)=k F_{1}\left(\tilde{Z}_{i j}\right)+(1-k) F_{2}\left(\tilde{Z}_{i j}\right), k \in[0,1],
$$

where:

$$
\begin{aligned}
& F_{1}\left(\tilde{Z}_{i j}\right)=a_{i j}^{1}+\frac{\left(a_{i j}^{4}-a_{i j}^{1}\right)+\left(a_{i j}^{3}-a_{i j}^{2}\right)}{2} \times \frac{1}{\sqrt{\left(a_{i j}^{4}-a_{i j}^{3}\right)^{2}+1}}, \\
& F_{2}\left(\tilde{Z}_{i j}\right)=a_{i j}^{4}-\frac{\left(a_{i j}^{4}-a_{i j}^{1}\right)+\left(a_{i j}^{3}-a_{i j}^{2}\right)}{2} \times \frac{1}{\sqrt{\left(a_{i j}^{2}-a_{i j}^{1}\right)^{2}+1}} .
\end{aligned}
$$

Since assessments are already real numbers, it comes to a classical decisionmaking problem. If criteria are of equal importance, aggregation operators without weighting coefficients (ATOKRI2 with matrices rows) are used. If weights of criteria are different real numbers, then either aggregation operators of ATOKRI1 or ATOKRI2 with matrix columns are used. If criteria weights are different real functions, ATOKRIF is used to aggregate the scores according to different criteria. Then the next steps of these algorithms are proceeded (see Fig. 2).

ARAKRI2 does not calculate fuzzy number indices to obtain aggregate estimates, and aggregated fuzzy numbers are obtained using direct aggregators and operations between fuzzy numbers. The first steps are the same as in ARAKRI1, and the index matrix of unified and normalized fuzzy numbers of (17) is used to determine the maximum and minimum fuzzy number of a given series. If criteria weighting are not set, aggregation operators without weighting coefficients (ATOKRI2) are used. If the weighting coefficients are real numbers, they are normalized and then either aggregation operators with weighting coefficients (ATOKRI1) or aggregation operators without weighting coefficients are used, but the fuzzy numbers are multiplied by the corresponding weights (ATOKRI2). If weighting functions are specified, ATOKRIF with matrices rows is used (see Fig. 2). As a result, for each alternative aggregated fuzzy numbers are obtained, which should be ranked in descending order. For this purpose, the indices (18) are calculated. The ranking of alternatives correspond to the ranking of indices.

Case 9. Criteria evaluations - fuzzy numbers and weighting coefficients are real functions

All criteria set fuzzy numbers, i.e., $m_{3}=m$ and criteria weights are different real functions $f_{1}(x), \ldots, f_{m}(x), x \in[0,1]-$ ARAKRI1 and/or ARAKRI2 are used, then ATOKRIF is used (see Fig. 2).

Case 10. Criteria and weighting coefficients - different

The criteria are different, i.e., $m=m_{1}+m_{2}+m_{3}$. ATOKRI1 is used for $m_{1}$ criteria, MMTRR for $m_{2}$ criteria, and ARAKRI3 for $m_{3}$ criteria. Since these algorithms reduce initial information to fuzzy preference relations depending on weighted coefficients of criteria, the task is reduced to one of the Cases 5-7. 
With ARAKRI3, the set of alternatives has been evaluated by various criteria, e.g., quantitative (utility functions), qualitative (crisp, nonfuzzy rankings), fuzzy relations, fuzzy numbers. It is necessary to bring this information to a common scale. A basic approach is to obtain fuzzy relations from available data by comparing the evaluations of alternatives pairwise for each criterion. In order to unify information, fuzzy relations on these criteria must be obtained. Using the index (18) it is possible to compare each pair of fuzzy numbers to obtain a membership degree of fuzzy relation. The ARAKRI1 algorithm indexes (18) from the matrix with unified and normalized fuzzy numbers is used. Then for each pair of alternatives $a_{i}, a_{j}$ and criteria is calculated the following value:

$$
\mu_{k}\left(a_{i}, a_{j}\right)=0.5+\frac{F\left(\tilde{Z}_{i k}\right)-F\left(\tilde{Z}_{j k}\right)}{2\left(F_{k}^{\max }-F_{k}^{\min }\right)}
$$

where $F_{k}^{\max }, F_{k}^{\min }$ are indices of the largest and smallest fuzzy number of the fuzzy numbers series for $k$-th criteria.

Thus, for each criterion whose evaluations are fuzzy numbers, a fuzzy relation of type (1) is obtained. This fuzzy relation is used in calculations of ATOKRI.

The solution of Task 3 is to analyse the rankings received in Task 2 and to select SMEs to be included in the cluster structure (see Fig. 1).

\section{Testing and analysis}

The following case study is considered: Technological Network (TN) with five nods ( 1 - manufacturers, 2 - suppliers, 3 - processors, 4 - research units, 5 -merchants); 25 enterprises selected in groups of five for each node; element of TN described with 14 criteria (Table 3). The list presented in Table 3 is an example. A define different set of criteria could be defined.

Table 3. List of criteria "Passport"

\begin{tabular}{|r|l|l|}
\hline No & Notation & Description \\
\hline 1 & $\left(k_{1}\right)$ & Export orientation index $=$ Export Vol. /Vol. of marketed production \\
\hline 2 & $\left(k_{2}\right)$ & Profitability $>0$ \\
\hline 3 & $\left(k_{3}\right)$ & General Liquidity ratio $\geq 1$ Financial independence $\left(k_{4}\right) \geq 0.67$ \\
\hline 4 & $\left(k_{4}\right)$ & Financial independence $\geq 0.67$ \\
\hline 5 & $\left(k_{5}\right)$ & Solvency \\
\hline 6 & $\left(k_{6}\right)$ & $\begin{array}{l}\text { Profitability }\{\text { Recommended thresholds: Node } 1>0.05 ; \text { Node } 2>0.1 ; \\
\text { Node } 3>0.14 ; \text { Node } 4>0.2 ; \text { Node } 5>0.25\}\end{array}$ \\
\hline 7 & $\left(k_{4}\right)$ & General Liquidity ratio $(\mathrm{k} 3) \geq 1$ Financial independence $\geq 0.67$ \\
\hline 8 & $\left(k_{8}\right)$ & Load capacity of the installed capacity => expert assessment \\
\hline 9 & $\left(k_{9}\right)$ & Predictive evaluation for marked extending => expert assessment \\
\hline 10 & $\left(k_{10}\right)$ & Sustainability Index of Research Institute Relations => expert assessment \\
\hline 11 & $\left(k_{11}\right)$ & Technological Development Index => expert assessment \\
\hline 12 & $\left(k_{12}\right)$ & Innovation Activity Index => Investment costs/Operation costs \\
\hline 13 & $\left(k_{13}\right)$ & $\begin{array}{l}\text { Index of investment activity in human resources = Inv. in human resource } \\
\text { /Operation costs }\end{array}$ \\
\hline 14 & $\left(k_{14}\right)$ & Infrastructure Index => expert assessment \\
\hline
\end{tabular}


Required input data:

1. Assessing of alternatives by criteria. Assessments refer to crisp criteria, fuzzy relations, and fuzzy numbers;

2. Values of possible weighting coefficients of criteria:

- real numbers $w_{1}, \ldots, w_{j}, \ldots, w_{m}$, where $w_{j}, j=1, \ldots, m$;

- parameters of real functions $f_{1}(x), \ldots, f_{m}(x), x \in[0,1]$, where $f_{j}(x)$, $j=1, \ldots, m$, are functions of types (13), (14), (15);

- the relation matrix of preference $W$ of type (16).

Since the calculation procedure is identical, here are presented the data only for Node 3 (Processors).

The Assessments of criteria presented as crisp numbers are given in Table 4. The first column contains identification of the assessed alternative. The first row shows the number of evaluated criterion, as shown in Table 3.

Table 4. Criteria assessments - crisp numbers

\begin{tabular}{|c|c|c|c|c|c|c|c|c|c|c|c|c|c|c|}
\hline Identification & $k_{1}$ & $k_{2}$ & $k_{3}$ & $k_{4}$ & $k_{5}$ & $k_{6}$ & $k_{7}$ & $k_{8}$ & $k_{9}$ & $k_{10}$ & $k_{11}$ & $k_{12}$ & $k_{13}$ & $k_{14}$ \\
\hline 3.1 & 0.500 & 0.600 & 2.286 & 0.714 & 1.579 & 1.600 & 0.050 & 4 & 4 & 3 & 4 & 0.400 & 0.030 & 4 \\
\hline 3.2 & 0.462 & 0.583 & 1.800 & 0.662 & 1.364 & 2.167 & 0.027 & 3 & 3 & 4 & 4 & 0.417 & 0.042 & 6 \\
\hline 3.3 & 0.467 & 0.500 & 2.111 & 0.661 & 1.592 & 1.875 & 0.037 & 5 & 4 & 4 & 4 & 0.388 & 0.048 & 5 \\
\hline 3.4 & 0.398 & 0.323 & 3.117 & 0.686 & 1.817 & 2.542 & 0.032 & 4 & 5 & 5 & 5 & 0.287 & 0.034 & 6 \\
\hline 3.5 & 0.352 & 0.244 & 2.707 & 0.885 & 3.240 & 2.346 & 0.032 & 4 & 6 & 4 & 5 & 0.298 & 0.025 & 7 \\
\hline
\end{tabular}

The scores of fuzzy criteria number of the type $\tilde{A}_{i j}=\left(a_{i j}^{1}, a_{i j}^{2}, a_{i j}^{3}, a_{i j}^{4}\right)$, $i=1, \ldots, n, j=1, \ldots, m$, where $a_{i j}^{1} \leq a_{i j}^{2} \leq a_{i j}^{3} \leq a_{i j}^{4}$ are real numbers.

In Tables 5, 6, 7 and 8 are presented assessments for $a_{i j}^{1}, a_{i j}^{2}, a_{i j}^{3}, a_{i j}^{4}$.

Table 5. Assessments for $a_{i j}^{1}$

\begin{tabular}{|c|c|c|c|c|c|c|c|c|c|c|c|c|c|c|}
\hline $\begin{array}{c}\text { Identifi- } \\
\text { cation }\end{array}$ & 1 & 2 & 3 & 4 & 5 & 6 & 7 & 8 & 9 & 10 & 11 & 12 & 13 & 14 \\
\hline 3.1 & 0.148 & 0.356 & 0.486 & 0.053 & 0.215 & 0.000 & 0.023 & 3 & 3 & 2 & 3 & 0.113 & 0.005 & 3 \\
\hline 3.2 & 0.110 & 0.339 & 0.000 & 0.001 & 0.000 & 0.567 & 0.000 & 2 & 2 & 3 & 3 & 0.130 & 0.017 & 5 \\
\hline 3.3 & 0.115 & 0.256 & 0.311 & 0.000 & 0.228 & 0.275 & 0.010 & 4 & 3 & 3 & 3 & 0.101 & 0.023 & 4 \\
\hline 3.4 & 0.046 & 0.079 & 1.317 & 0.025 & 0.453 & 0.942 & 0.005 & 3 & 4 & 4 & 4 & 0.000 & 0.009 & 5 \\
\hline 3.5 & 0.000 & 0.000 & 0.907 & 0.224 & 1.876 & 0.746 & 0.005 & 3 & 5 & 3 & 4 & 0.011 & 0.000 & 6 \\
\hline
\end{tabular}

Table 6. Assessments for $a_{i j}^{2}$

\begin{tabular}{|c|c|c|c|c|c|c|c|c|c|c|c|c|c|c|}
\hline $\begin{array}{c}\text { Identifi- } \\
\text { cation }\end{array}$ & 1 & 2 & 3 & 4 & 5 & 6 & 7 & 8 & 9 & 10 & 11 & 12 & 13 & 14 \\
\hline 3.1 & 0.58 & 0.81 & 2.89 & 0.77 & 2.13 & 2.11 & 0.06 & 5 & 5 & 4 & 5 & 0.47 & 0.04 & 5 \\
\hline 3.2 & 0.55 & 0.79 & 2.40 & 0.72 & 1.92 & 2.67 & 0.04 & 4 & 4 & 5 & 5 & 0.49 & 0.05 & 7 \\
\hline 3.3 & 0.55 & 0.71 & 2.72 & 0.72 & 2.15 & 2.38 & 0.05 & 6 & 5 & 5 & 5 & 0.46 & 0.06 & 6 \\
\hline 3.4 & 0.48 & 0.53 & 3.72 & 0.75 & 2.37 & 3.05 & 0.04 & 5 & 6 & 6 & 6 & 0.36 & 0.04 & 7 \\
\hline 3.5 & 0.44 & 0.45 & 3.31 & 0.95 & 3.79 & 2.85 & 0.04 & 5 & 7 & 5 & 6 & 0.37 & 0.04 & 8 \\
\hline
\end{tabular}


Table 7. Assessments for $a_{i j}^{3}$

\begin{tabular}{|c|c|c|c|c|c|c|c|c|c|c|c|c|c|c|}
\hline $\begin{array}{c}\text { Identifi- } \\
\text { cation }\end{array}$ & 1 & 2 & 3 & 4 & 5 & 6 & 7 & 8 & 9 & 10 & 11 & 12 & 13 & 14 \\
\hline 3.1 & 1.46 & 1.71 & 7.70 & 2.22 & 5.97 & 6.32 & 0.1298 & 6 & 6 & 5 & 6 & 1.187 & 0.1124 & 6 \\
\hline 3.2 & 1.42 & 1.69 & 7.21 & 2.17 & 5.76 & 6.89 & 0.1068 & 5 & 5 & 6 & 6 & 1.204 & 0.1244 & 8 \\
\hline 3.3 & 1.42 & 1.61 & 7.52 & 2.16 & 5.98 & 6.59 & 0.1168 & 7 & 6 & 6 & 6 & 1.175 & 0.1304 & 7 \\
\hline 3.4 & 1.35 & 1.43 & 8.53 & 2.19 & 6.21 & 7.26 & 0.1118 & 6 & 7 & 7 & 7 & 1.074 & 0.1164 & 8 \\
\hline 3.5 & 1.31 & 1.35 & 8.12 & 2.39 & 7.63 & 7.06 & 0.1118 & 6 & 8 & 6 & 7 & 1.085 & 0.1074 & 8 \\
\hline
\end{tabular}

Table 8. Assessments for $a_{i j}^{4}$

\begin{tabular}{|c|c|c|c|c|c|c|c|c|c|c|c|c|c|c|}
\hline $\begin{array}{l}\text { Identifi- } \\
\text { cation }\end{array}$ & 1 & 2 & 3 & 4 & 5 & 6 & 7 & 8 & 9 & 10 & 11 & 12 & 13 & 14 \\
\hline 3.1 & 1.891 & 2.156 & 10.103 & 2.939 & 7.889 & 8.424 & 0.165 & 7 & 7 & 6 & 7 & 1.545 & 0.148 & 7 \\
\hline 3.2 & 1.853 & 2.139 & 9.617 & 2.887 & 7.674 & 8.991 & 0.142 & 6 & 6 & 7 & 7 & 1.562 & 0.160 & 8 \\
\hline 3.3 & 1.858 & 2.056 & 9.928 & 2.886 & 7.902 & 8.699 & 0.152 & 8 & 7 & 7 & 7 & 1.533 & 0.166 & 8 \\
\hline 3.4 & 1.789 & 1.879 & 10.934 & 2.911 & 8.127 & 9.366 & 0.147 & 7 & 8 & 8 & 8 & 1.432 & 0.152 & 8 \\
\hline 3.5 & 1.743 & 1.800 & 10.524 & 3.110 & 9.550 & 9.170 & 0.147 & 7 & 8 & 7 & 8 & 1.443 & 0.143 & 8 \\
\hline
\end{tabular}

Criteria Assessments as fuzzy relations are calculated by a matrix of fuzzy relation of weights. The values for $\alpha, \beta$ and $\gamma$ and the fuzzy relations for weighting coefficients used for all five Nodes are presented in Tables 9 and 10. Equal crisp weighting coefficients are introduced for all criteria.

Table 9. Values for $\alpha, \beta, \gamma$

\begin{tabular}{|c|c|c|c|c|c|c|c|c|c|c|c|c|c|c|}
\hline $\begin{array}{c}\text { Coefi- } \\
\text { cient }\end{array}$ & \multicolumn{10}{|c|}{ Criteria } \\
\cline { 2 - 15 } & 1 & 2 & 3 & 4 & 5 & 6 & 7 & 8 & 9 & 10 & 11 & 12 & 13 & 14 \\
\hline$\alpha$ & 0.1 & 0.150 & 0.020 & 0.10 & 0.12 & 0.080 & 0.06 & 0.015 & 0.080 & 0.090 & 0.09 & 0.015 & 0.015 & 0.065 \\
\hline$\beta$ & 0.1 & 0.080 & 0.060 & 0.05 & 0.05 & 0.045 & 0.02 & 0.015 & 0.018 & 0.012 & 0.10 & 0.130 & 0.150 & 0.170 \\
\hline$\gamma$ & 0.2 & 0.150 & 0.045 & 0.01 & 0.01 & 0.015 & 0.15 & 0.170 & 0.090 & 0.040 & 0.02 & 0.030 & 0.045 & 0.025 \\
\hline
\end{tabular}

Table 10. Weighting fuzzy relation values

\begin{tabular}{|c|c|c|c|c|c|c|c|c|c|c|c|c|c|c|}
\hline Weigh- & \multicolumn{14}{|c|}{ Weighted relations } \\
\hline \multirow{14}{*}{$W$} & 0.5 & 0.6 & 0.3 & 0.2 & 0.8 & 0.2 & 0.3 & 0.6 & 0.8 & 0.4 & 0.5 & 0.3 & 0.2 & 0.4 \\
\hline & 0.4 & 0.5 & 0.8 & 0.8 & 0.3 & 0.6 & 0.7 & 0.5 & 0.3 & 0.6 & 0.9 & 0.7 & 0.6 & 0.4 \\
\hline & 0.7 & 0.2 & 0.5 & 0.4 & 0.6 & 0.4 & 0.8 & 0.7 & 0.9 & 0.7 & 0.6 & 0.4 & 0.5 & 0.3 \\
\hline & 0.8 & 0.2 & 0.6 & 0.5 & 0.6 & 0.4 & 0.4 & 0.4 & 0.8 & 0.7 & 0.6 & 0.6 & 0.8 & 0.4 \\
\hline & 0.2 & 0.7 & 0.4 & 0.4 & 0.5 & 0.3 & 0.5 & 0.6 & 0.2 & 0.3 & 0.4 & 0.8 & 0.6 & 0.7 \\
\hline & 0.8 & 0.4 & 0.6 & 0.6 & 0.7 & 0.5 & 0.3 & 0.2 & 0.7 & 0.4 & 0.6 & 0.5 & 0.4 & 0.7 \\
\hline & 0.5 & 0.3 & 0.2 & 0.6 & 0.5 & 0.7 & 0.5 & 0.3 & 0.4 & 0.2 & 0.7 & 0.8 & 0.7 & 0.5 \\
\hline & 0.7 & 0.5 & 0.3 & 0.6 & 0.4 & 0.8 & 0.7 & 0.5 & 0.4 & 0.4 & 0.6 & 0.5 & 0.8 & 0.5 \\
\hline & 0.4 & 0.7 & 0.1 & 0.2 & 0.8 & 0.3 & 0.6 & 0.6 & 0.5 & 0.2 & 0.3 & 0.5 & 0.4 & 0.9 \\
\hline & 0.2 & 0.4 & 0.3 & 0.3 & 0.7 & 0.6 & 0.8 & 0.6 & 0.8 & 0.5 & 0.4 & 0.5 & 0.5 & 0.8 \\
\hline & 0.5 & 0.1 & 0.4 & 0.4 & 0.6 & 0.4 & 0.3 & 0.4 & 0.7 & 0.6 & 0.5 & 0.6 & 0.7 & 0.4 \\
\hline & 0.7 & 0.3 & 0.6 & 0.4 & 0.2 & 0.5 & 0.2 & 0.5 & 0.5 & 0.5 & 0.4 & 0.5 & 0.4 & 0.3 \\
\hline & 0.8 & 0.4 & 0.5 & 0.2 & 0.4 & 0.6 & 0.3 & 0.2 & 0.6 & 0.5 & 0.3 & 0.6 & 0.5 & 0.2 \\
\hline & 0.6 & 0.6 & 0.7 & 0.6 & 0.3 & 0.3 & 0.5 & 0.5 & 0.1 & 0.2 & 0.6 & 0.7 & 0.8 & 0.5 \\
\hline
\end{tabular}


The descending rankings of enterprises by five Nodes are presented in Tables 11 and 12. The first columns contain the names of algorithms. The second column shows weighted coefficients. The last columns present the ranking. Numbers 1 through 5 are enterprises' identifiers in a given node, with the entire rank derived from algorithm presented in the corresponding rows.

Table 11. Fuzzy algorithms ranking for Node 1, Node 2 and Node 3

\begin{tabular}{|l|l|l|l|l|l|l|l|l|l|l|l|l|l|l|l|l|}
\hline Algorithm & Weights & \multicolumn{3}{|c|}{ Node 1} & \multicolumn{3}{|c|}{ Node 2} & \multicolumn{5}{|c|}{ Node 3} \\
\hline ATOKRI 1 & W mean & 3 & 2 & 5 & 1 & 4 & 3 & 4 & 5 & 2 & 1 & 4 & 5 & 3 & 1 & 2 \\
\hline ATOKRI 2 & MaxMin & 3 & 2 & 4 & 5 & 1 & 3 & 4 & 5 & 1 & 2 & 3 & 4 & 5 & 2 & 1 \\
\hline ATOKRI 2 & MinAvg & 2 & 3 & 5 & 4 & 1 & 4 & 5 & 2 & 3 & 1 & 5 & 4 & 1 & 3 & 2 \\
\hline ARAKRI MaxMin ATOKRI & MinAvg & 2 & 3 & 5 & 1 & 4 & 3 & 4 & 5 & 2 & 1 & 5 & 4 & 1 & 3 & 2 \\
\hline ARAKRI MaxProd ATOKRI & MinAvg & 2 & 3 & 5 & 1 & 4 & 3 & 4 & 5 & 2 & 1 & 4 & 5 & 1 & 3 & 2 \\
\hline ATOKRIF & Linear & 2 & 3 & 5 & 4 & 1 & 3 & 4 & 5 & 2 & 1 & 3 & 4 & 5 & 1 & 2 \\
\hline ATOKRIF & ParLinear & 2 & 3 & 5 & 1 & 4 & 3 & 4 & 5 & 1 & 2 & 5 & 4 & 2 & 3 & 1 \\
\hline ATOKRIF & Quadratic & 2 & 3 & 5 & 4 & 1 & 3 & 4 & 5 & 2 & 1 & 3 & 4 & 5 & 1 & 2 \\
\hline AARAKRI 2 & MaxMin & 4 & 2 & 3 & 5 & 1 & 5 & 1 & 2 & 3 & 4 & 5 & 4 & 3 & 2 & 1 \\
\hline AARAKRI 2 & MinAvg & 3 & 2 & 5 & 4 & 1 & 5 & 4 & 3 & 2 & 1 & 4 & 5 & 3 & 2 & 1 \\
\hline AARAKRI 2 & Gamma & 3 & 2 & 5 & 4 & 1 & 5 & 3 & 4 & 2 & 1 & 5 & 4 & 3 & 2 & 1 \\
\hline
\end{tabular}

For Node 1 (Table 11), ATOKRI algorithms rank as leading enterprises 3, 2, and 5 ( 1 and 4 may be excluded from further consideration). ARAKRI algorithms specify enterprises 2,3 and 5 (5 and 4 may be excluded). ATOKRIF algorithms give the same ranking for the first three positions $-2,3$ and 5 . The results form AARAKRI2 algorithm also indicates 3, 2 and 5 ranked on top three. Finally, it can be noted that the algorithms give close ranking for first places (enterprises 2, 3 and 5).

For Node 2 (Table 11) the ATOKRI refers to enterprises 3, 4, and 5 as top ranking ( 2 and 1 can be excluded). The ARAKRI specify 3,4 , and 5 as the leading ( 2 and 1 can be excluded from further consideration). The ATOKRIF gives the same ranking for the first three positions as 3,4 and 5. The AARAKRI refer to 5 as top of the list, but for the rest of the data ranking is different. Finally, fuzzy algorithms give almost identical solutions and rank enterprises 3, 4 and 5 on the top.

The ranking for Node 3 (Table 11) of ATOKRI algorithms show as leading enterprises 4, 5 and 3, and the offsetting in ranking is a result of different weighted coefficients. Enterprises 4, 5 and 3 can be included form the list ( 2 and 1 may be excluded). The algorithms ARAKRI indicate 5 and 4 as leading. For the third, fourth and fifth ranked enterprises algorithms give the same result respectively 1,3 and 2 . ATOKRIF algorithms give the first three positions to 3, 4 and 5. In essence, the AARAKRI algorithms give a similar result.

For Node 4 (Table 12) ATOKRI algorithms rank enterprises 2, 1 and 5 top positions, and the offset in ordering is a result of different weighting coefficients. ARAKRI algorithms give close ranking - the first three are 1, 2 and 4 . The ATOKRIF 
algorithms give the first three positions to 2,5 and 1, with the same ranking of the third position for 1 . AARAKRI2 gives the first position to 5 and the second to 2 . In conclusion, fuzzy algorithms give predominantly the first positions to 1,2 and 5 .

The ranking for Node 5 (Table 12) show an almost identical ranking for the top three to 4,1 and 5 .

The results obtained using fuzzy algorithms for selecting enterprises to fill TN can be summarized as follows: Node $1\{3,2,5\}$, Node $2\{3,4,5\}$, Node $3\{4,5,3\}$, Node $4\{1,2,5\}$, Node $5\{4,5,1\}$.

Table 12. Ranking of fuzzy algorithms for Node 4 and Node 5

\begin{tabular}{|l|l|l|l|l|l|l|l|l|l|l|l|}
\hline Algorithm & Weights & \multicolumn{5}{|c|}{ Node 4} & \multicolumn{5}{|c|}{ Node 5} \\
\hline ATOKRI 1 & W mean & 2 & 1 & 5 & 3 & 4 & 4 & 1 & 5 & 2 & 3 \\
\hline ATOKRI 2 & MaxMin & 1 & 4 & 5 & 2 & 3 & 4 & 1 & 5 & 2 & 3 \\
\hline ATOKRI 2 & MinAvg & 2 & 3 & 1 & 5 & 4 & 4 & 5 & 1 & 2 & 3 \\
\hline ARAKRI MaxMin ATOKRI & MinAvg & 1 & 2 & 4 & 5 & 3 & 4 & 1 & 5 & 2 & 3 \\
\hline ARAKRI MaxProd ATOKRI & MinAvg & 1 & 2 & 4 & 5 & 3 & 4 & 1 & 5 & 2 & 3 \\
\hline ATOKRIF & Linear & 2 & 5 & 1 & 3 & 4 & 4 & 1 & 5 & 2 & 3 \\
\hline ATOKRIF & ParLinear & 5 & 2 & 1 & 4 & 3 & 4 & 5 & 1 & 2 & 3 \\
\hline ATOKRIF & Quadratic & 2 & 5 & 1 & 3 & 4 & 4 & 1 & 5 & 2 & 3 \\
\hline AARAKRI 2 & MaxMin & 5 & 2 & 1 & 3 & 4 & 4 & 5 & 1 & 2 & 3 \\
\hline AARAKRI 2 & MinAvg & 1 & 3 & 5 & 4 & 2 & 4 & 5 & 1 & 2 & 3 \\
\hline AARAKRI 2 & Gamma & 5 & 2 & 4 & 3 & 1 & 4 & 5 & 1 & 2 & 3 \\
\hline
\end{tabular}

For verification the above results are compared to rankings obtained by PROMETHEE II outranking method shown in Table 13.

Table 13. PROMETHEE II ranking of Nodes 1, 2, 3, 4 and 5

\begin{tabular}{|c|c|c|c|c|c|c|c|c|c|}
\hline \multicolumn{2}{|c|}{ Node 1} & \multicolumn{2}{c|}{ Node 2} & \multicolumn{2}{c|}{ Node 3} & \multicolumn{2}{c|}{ Node 4} & \multicolumn{2}{c|}{ Node 5} \\
\hline Ident. & $\Phi_{1}$ & Ident. & $\Phi_{2}$ & Ident. & $\Phi_{3}$ & Ident. & $\Phi_{4}$ & Ident. & $\Phi_{5}$ \\
\hline $\mathbf{1 . 3}$ & $\mathbf{1 . 0 5 3 5}$ & $\mathbf{2 . 4}$ & $\mathbf{1 . 1 1 7 5}$ & $\mathbf{3 . 4}$ & $\mathbf{0 . 6 9 5 9}$ & $\mathbf{4 . 1}$ & $\mathbf{0 . 1 3 4 4}$ & $\mathbf{5 . 4}$ & $\mathbf{1 . 6 5 7 3}$ \\
\hline $\mathbf{1 . 2}$ & $\mathbf{0 . 4 6 6 2}$ & $\mathbf{2 . 3}$ & $\mathbf{0 . 4 1 8 6}$ & $\mathbf{3 . 5}$ & $\mathbf{0 . 6 4 4 6}$ & $\mathbf{4 . 2}$ & $\mathbf{0 . 0 5 2 4}$ & $\mathbf{5 . 5}$ & $\mathbf{0 . 5 6 1 6}$ \\
\hline $\mathbf{1 . 5}$ & $\mathbf{0 . 0 9 8 7}$ & $\mathbf{2 . 5}$ & $\mathbf{0 . 2 7 2 5}$ & 3.3 & -0.0956 & $\mathbf{4 . 5}$ & $\mathbf{0 . 0 2 6 5}$ & 5.1 & 0.4032 \\
\hline 1.4 & -0.2696 & 2.2 & -0.2192 & 3.1 & -0.3735 & 4.3 & -0.147 & 5.2 & -0.7616 \\
\hline 1.1 & -1.4764 & 2.1 & -1.37 & 3.2 & -0.8563 & 4.4 & -0.1601 & 5.3 & -1.5932 \\
\hline
\end{tabular}

The results are summarized as follow: Node $1:\{\mathbf{3}, \mathbf{2}, \mathbf{5}, 4,1\}$, Node $2:\{\mathbf{4}, \mathbf{3}, \mathbf{5}$, 2, 1\}, Node 3: $\{\mathbf{4}, \mathbf{5}, \mathbf{3}, 1,2\}$, Node $4:\{\mathbf{1}, \mathbf{2}, \mathbf{5}, 3,4\}$ and Node $5:\{\mathbf{4}, \mathbf{5}, 1,2,3\}$.

As the whole, it can be assumed that the results obtained from fuzzy algorithms and those of PROMETHEE II coincide. The small shifts in the ranking are most likely due to differences in input data. This comparison is important in that, in the absence of significant differences, the use of fuzzy algorithms gives more freedom in selection and precision of input data and some inaccuracies do not affect the quality of the recommended solutions. PROMETHEE II is a classical method that has proven its 
working ability, but it works with accurate data, which due to the specifics of the task is not always possible. The confirmed results show that a fuzzy algorithm approach would find its place in solving the task of economic clustering.

\section{Conclusion}

The presented paper proposes an approach to solving a problem for selection of enterprises - potential participants in economic cluster using fuzzy sets theory methods. The approach implementation is illustrated by an example performed by fuzzy algorithms with results compared to PROMETHEE II multi-criteria outranking method. The test confirms the concept that, in solving application problems, the use of fuzzy algorithms gives a good quality solution.

From the test shown, fuzzy algorithms require significantly more complex input information, which can be interpreted as a difficulty. In this regard, it is appropriate to clarify the specific conditions that would require preferring fuzzy algorithms to classical ones. The answer should be sought in the terms of problem formulation, which initially is defined as a "poorly structured problem under uncertainty", because the goal is to design a cluster structure from enterprises where it is difficult to set a clear target function. Presumably, the enterprise management models, including economic clustering are burdened with poorly structured information that makes it difficult to implement by traditional decision-support methods. This means that the development of such technologies requires this factor to be taken into account.

One of the advantages of the proposed approach is that it is autonumouse to decision-making tools used. This allows future development and improvement to be sought in the application of a variety of methods in domain of soft computing area [1]. As a further development it could be considered experiments with additional fuzzy algorithms for group a priory multi-criteria decision making such as TOPSIS method and a combination of two fuzzy methods by applying IT2FN - DEMATEL and VIKOR. TOPSIS method is a compensatory method that provides more realistic modelling of multi-criteria analysis task compering to non-compensatory methods $[8,9]$. Another line in future development could be fined in the implementation of InterCriteria Analysis (ICA) method. It offers a new way in comparison of individual criteria out of set of criteria by which a set of objects are evaluated [2, 14, 29].

Fuzzy algorithms give freedom in a way that input data is set, and in particular do not require accurate estimates. Given the subject of reaches - economic clustering, the problem of availability of accurate data is serious due to insufficient historical data available.

Acknowledgements: The author is thankful for the support provided by the Bulgarian National Science Fund under Grand No DFNI-I-02-5 "InterCtriteria Analysis: A New Approach to Decision Making". 


\section{References}

1. A n g e lov a, V. Investigations in the Ares of Soft Computing. Targeted State of the Art Report. Cybernetics and Information Technologies, Vol. 9, 2009, No 1, pp. 18-24. http://www.cit.iit.bas.bg/CIT_09/v9-1/18-24.pdf

2. A t a n a s s ova, V., L. D o u k ov sk a, A. M i ch a lík ová, I. R a d e va. Intercriteria Analysis: From Pairs to Triples. - Notes on Intuitionistic Fuzzy Sets, Vol. 22, 2016, No 5, pp. 98-110.

3. Brans, J. P., B. Mareschal. The PROMCALC\&GAIA Decision Support System for Multicriteria Decision Aid. - Decision Support Systems, North-Holland, Vol. 12, 1994, pp. 279-310.

4. Copland, T., T. Koller, J. Murrin. Valuation: Measure and Managing the Value of Companies. New York, John Wiley, 2002.

5. K i s h o r, D. R., N. B. V e n k a t e s w a r l u. Hybridization of Expectation-Maximization and K-Means Algorithms for Better Clustering Performance. - Cybernetics and Information Technologies, Vol. 16, 2016, No 2, pp. 16-34

6. I 1 i e v a, G. Decision Making Methods in Agent Based Modeling. - In: Proc. of Workshop on Applications of Software Agents, 2011, pp. 8-17.

7. I li e va, G. A Fuzzy Approach for Bidding Strategy Selection. - Cybernetics and Information Technologies, Vol. 12, 2012, No 1, pp. 61-69.

8. Il i e va, G. TOPSIS Modification with Interval Type-2 Fuzzy Numbers. - Cybernetics and Information Technologies, Vol. 16, 2016, No 2, pp. 60-68.

9. I 1 i e v a, G. Group Decision Analysis with Interval Type-2 Fuzzy Numbers. - Cybernetics and Information Technologies, Vol. 17, 2017, No 1, pp. 31-44.

10. G e or gi e va, P., I. P o p ch e v. Application of Q-Measure in a Real Time Fuzzy System for Managing Financial Assets. - International Journal of Soft Computing (IJSC), Vol. 3, 2012, No 4, pp. 21-38.

11. G e or g i e v a, P., I. P o p c h e v, S. S t o y a n o v. A Multi-Step Procedure for Asset Allocation in Case of Limited Resources. - Cybernetics and Information Technologies, Vol. 15, 2015, No 3, pp. 41-51.

12. G h a z a n f a r i, M., S. R o u h a n i, M. J a f a ri. A Fuzzy TOPSIS Model to Evaluate the Business Intelligence Competencies of Port Community Systems. - Polish Maritime Research, Vol. 2, Vol. 21, 2014, No 82, pp. 86-96.

13. Herrera-Vi e d m a, E., Herrer a, F. Ch i c l a n a, M. L u qu e. Some Issues on Consistency of Fuzzy Preference Relations. - European Journal of Operational Research, 2004, pp. 98-109.

14. M a v rov, D., I. R a d e va, K. A t a n a s o v, L. D o u k o v s k a, I. K a l a y k o v. Inter Criteria Software Design: Graphic Interpretation within the Intuitionistic Fuzzy Triangle. - In: Proc. of International Symposium on Business Modeling and Software Design (BMSD'15), Milan, Italy, SCITEPRESS - Science and Technology Publications, 2015, pp. 279-283.

15. P o p c h e v, I., V. P e n e v a. An Algorithm for Comparison of Fuzzy Sets. - Fuzzy Sets and Systems, Norht-Holland, Amsterdam, Vol. 60, 1993, No 1, pp. 59-65.

16. Peneva, V., I. Popchev. Fuzzy Ordering on the Base of Multicriteria Aggregation. Cybernetics and Systems, Vol. 29, 1998, No 6, pp. 613-623.

17. P e n e v a, V., I. P o p c h e v. Fuzzy Logic Operators in Decision-Making. - International Journal Cybernetics and Systems, Vol. 30, 1999, No 8, pp. 725-745.

18. P e n e va, V., I. P o p ch e v. Aggregation on Fuzzy Numbers in a Decision Making Situation. Cybernetics and Systems, Vol. 32, 2001, Issue 8, pp. 871-885.

19. P e n e v a, V., I. P o p c h e v. Aggregation of Fuzzy Relations Using Weighting Function. - Compt. Rend. Acad. bulg. Sci., Vol. 60, 2007, No 10, pp. 1047-1052.

20. P e n e v a, V., I. P o p c h e v. Fuzzy Criteria Importance with Weighting Functions. - Comp. Rend. Acad. bulg. Sci. Vol. 61, 2008, No 3, pp. 293-300.

21. P e n e va, V., I. P o p che v. Models for Fuzzy Multicriteria Decision Making Based on Fuzzy Relations. - Compt. Rend. Acad. bulg. Sci., Vol. 62, 2009, No 5, pp. 551-558.

22. P e n e va, I., I. P o p c h e v. Fuzzy Multi-Criteria Decision Making Algorithms. - Comp. Rend. Acad. bulg. Sci., Vol. 63, 2010, No 7, pp. 979-991. 
23. P o p c h e v, I., I. R a d e v a. MAP-Cluster: An Approach for Latent Cluster Identification. - In: Proc. of Synergy of Computational Economics and Financial and Industrial Systems IFAC CEFIS'2007, 2007, Istanbul, pp. 63-67.

24. Porter, M. On Competition, Clusters and Competition: New Agendas for Companies, Governments, and Institutions. Boston, Harvard Business School Press, 1998.

25. $\mathrm{R}$ a d e v a, I. An Approach to Strategic Integration in Economic Clustering. - In: International Conference Automatics and Informatics'10, Sofia, 2010, pp. II-385-388.

26. R a d e v a, I. Strategic Integration with MAP - CLUSTER Software System. - Cybernetics and Information Technologies, Vol. 10, 2010, No 2, pp.78-93.

27. R o uh an i, S., M. Gha z a $\mathrm{f}$ ar i, M. J a f ari. Evaluation Model of Business Intelligence for Enterprise Systems Using Fuzzy TOPSIS. - Expert Systems with Applications, Vol. 39, 2012, pp. 3764-3771.

28. S z m i d t, E., J. K a c pr z y k, K. A t a n a s s o v. Intuitionistic Fuzzy Modifications of Some Peneva-Popchev Formulas for Estimation of Preference Degree. Pary 1. - In: Issue in IFSs and GNs, Vol. 10, 2013, pp. 12-20.

29. InterCriteria Research Portal.

http://www.intercriteria.net/publications 